\title{
A metastatic tumor is no different to a viral pandemic: lessons learnt from COVID-19 may teach us to change the PRRT paradigm
}

\author{
Giovanni Paganelli ${ }^{1} \cdot$ Lisa Bodei $^{2} \cdot$ Irvin Modlin ${ }^{3,4}$ \\ Published online: 20 June 2020 \\ (C) Springer-Verlag GmbH Germany, part of Springer Nature 2020
}

Keywords COVID $\cdot$ FDG-PET $\cdot$ NETest $\cdot$ Neuroendocrine tumor $\cdot$ PPQ $\cdot$ PRRT

Peptide Receptor RadioTherapy (PRRT) came of age with the first official announcement in 2015 when the results of the NETTER-1 randomized trial $[1,2]$ became available. This demonstrated that second-line therapy with $\left[{ }^{177} \mathrm{Lu}\right] \mathrm{Lu}-$ DOTA-TATE was significantly more efficient than highdose somatostatin analogs (SSAs) in decreasing the risk of progression of metastatic small-bowel neuroendocrine tumors (NETs), receiving standard SSA dosage. Those of us who had already been using PRRT effectively for 20 years were underwhelmed by this pronouncement. It was like listening to the result of a football game being announced a week after we had watched the match.

Similarly, since 2003 we have known what viral pandemics like SARS can do to the population. Yet, almost 20 years later, we responded as if we had learnt nothing about early identification, predicting outcome and instituting early treatment. Much the same pattern has emerged with PRRT. We waited 25 years after the first demonstration of PRRT efficacy for there to be approval of $\left[{ }^{177} \mathrm{Lu}\right] \mathrm{Lu}$-DOTA-TATE as a pharmaceutical for PRRT [3-6]. In the meantime, the slow adopters, parties with vested interests and naysayers, hindered the usage of an obviously effective therapy. And now we are waiting for a predictor of outcome and for-dare one say it - the

This article is part of the Topical Collection on Oncology general

Irvin Modlin

imodlin@optonline.net

1 Nuclear Medicine Operative Unit, Istituto Scientifico Romagnolo per lo Studio e la Cura dei Tumori (IRST) IRCCS, 47014 Meldola, Italy

2 Department Nuclear Medicine, Memorial Sloan Kettering Cancer Center, New York, NY 10065, USA

3 School of Medicine, Yale University, New Haven, CT 06520, USA

4 Gastroenterological and Endoscopic Surgery, Yale University School of Medicine, New Haven, CT 06520-8062, USA opportunity to implement effective treatment early... before the tumor has spread through the entire body.

Relying on the results of the NETTER-1 study, the Medical Oncology and Endocrinological community has now embraced PRRT as if no previous data were relevant. Thus, none of those who have used $\left[{ }^{177} \mathrm{Lu}\right] \mathrm{Lu}$-DOTA-TATE in spontaneous single-arm academic trials for over 15 years will be surprised when the mature results demonstrating an overall survival benefit will be available.

Should we be happy that PRRT is now considered as second-line therapy in progressive metastatic GEP NET patients? Probably not, since many patients will have perished having waiting too long before receiving a targeted therapy with a safe and efficient drug. Watching and waiting as a strategy for COVID-19 and SARS proved to be a futile strategy to look after illness. In other environments it would be termed benign neglect and governments that have recommended inactivity have been justifiably criticized at the negative outcomes of such a policy. Indeed, it is a quixotic notion (inactivity) when considering a proliferating tumor; it will not get better on its own or are there some who believe such an improbable notion? Just as the three Ts - testing, tracing, and treating - have been well proven as a pandemic management strategy, so we need to re assess PRRT. Firstly, we need to test for and develop assessments of tumor-specific genetic drivers that identify progression. In addition, we need to identify the presence of the target (SSA-PET) (trace where the tumor is), define the activity, and treat the extent of the disease. In order to ensure effective treatment we need to characterize both the extent and the likely responsiveness of the NET disease (e.g., NETest and the positive predictor quotient (PPQ) $[7,8]$ ) and implement an early prognostic characterization of the disease $\left(\left[{ }^{18} \mathrm{~F}\right]\right.$-FDG PET/CT $\left.[9,10]\right)$. This should provide the basis for the implementation of early treatment (PRRT).

We understand that containment is effective for COVID19 , why would we not seek to contain a tumor at the earliest 
possible time before it spreads as a pandemic through the body? Should we have to wait for meaningful radiological progression, based upon RECIST criteria, with all its known limitations in NETs, before we can take action? Is there any biological proof that a tumor is in a state of suspended animation-static, non-metabolic, and non-proliferative?

If we compare this scenario to the current COVID-19 pandemic, with all due proportionality related to the different biological pace, the analogy would be that in addition to early signs of fever and cough, one should await respiratory failure before initiating an active treatment targeting the disease. In both cases-but more so for NETs, which are better understood - should we wait to have a much greater magnitude of progression, defined according to obsolescent schemes, and only treat when the outcome is inevitable? The equivalent would be to wait till the pandemic has moved to many different locations and incidence levels are now increasing in multiple sites before we propose to act. Surely, we are imprudent and negligent if we do not seek to move treatment time needle forwards to increase efficacy (Fig. 1).

Presently, major guidelines recommend surveillance even in asymptomatic metastatic cases of low-grade NETs with low tumor burden $[11,12]$. However, a recent meta-analysis indicates the superiority of active treatments over placebo, in terms of progression-free and overall survival [13]. As for COVID-19, it is clear that waiting for the disease to progress, in the single individual and in the community, before taking action, is like securing the cage door after the lion has escaped. The same is true for NET disease, and one should be aware of the risks of "tumor escape" and behave accordingly before the disease engulfs the victim.

The identification of the minimum effective dosage while maintaining maximum tolerability is a critical determinant in optimizing efficacy of PRRT [14] and, more generally, target therapies. If these could be used at early — even microscopic stages of disease, significantly better results could be obtained,

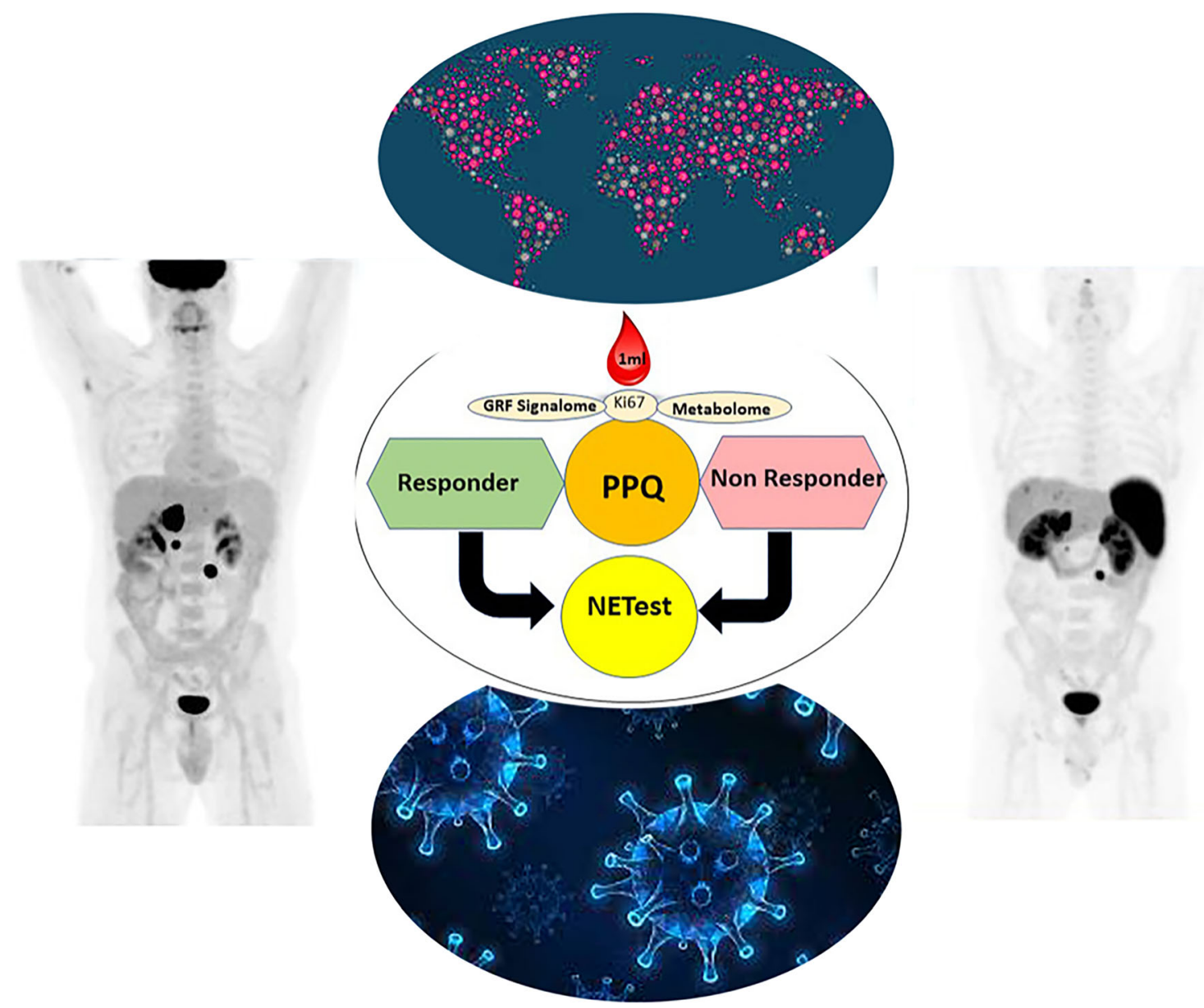

Fig. 1 Metastatic disease as a paradigm model of an intra-individual pandemic. COVID-19 (bottom) has metastasized throughout the countries of the world (top). Targeted therapy can be directed against NETs using a personalized metabolic functional imaging FDG PET (left) and SSA-PET (right) assessing glycolytic tumor metabolism and SSTR2 expression. The FDG PET ${ }^{68}$ demonstrates increased metabolic activity, and the SSA-PET (right) indicates the target-increased SSTR expression in the minimal metastatic disease. The efficacy of PRRT can then be predicted using molecular genomic analysis in blood (PPQ) (center) and monitored in real time using the NETest, avoiding excessive radiation from frequent reassessment $\mathrm{CT}$ scans. Fully anonymized images are from MSKCC 
similarly to adjuvant chemotherapy [15]. Treating minimal residual disease is, in fact, more effective than attempting to do so in advanced stages, with respect to disease volume, bioavailability and permeation of the drug into tumor cells, and acquired tumor damage/immune response/repair capacity [16].

A critical issue is to have sensitive non-invasive tools that identify residual/recurrent disease at the earliest possible time. These include molecular genomic liquid biopsies such as the NETest and advanced functional imaging [17]. In the same way that confronting COVID-19 would have been much more effective if an early notification test was available and we identified the first ten infected persons before they became multiplex global vectors. In precisely this fashion so would a tumor patient benefit if we could detect an early genomic disease signal and identify a tumor when only minimal aberrant clones commenced proliferation.

In reality, the efficacy of radionuclide therapy is determined by the balance between the absorbed radiation dose delivered to the tumor (Gy) - in turn regulated by the tumor mass, administered activity, and residence time - and the responsiveness of the tumor to radiation, which has a genomic basis [18].

The crux of the matter is to ensure that therapeutic procrastination should not be allowed to amplify disease burden, thereby placing patients at higher risk. Such a strategy facilitates the development of disease likely to be less responsive and simultaneously incurring the adverse events associated with ionizing radiation. PRRT should not be offered arbitrarily to any patient with a low-volume stable G1-G2 GEP-NET disease. A molecular and image integrated stratification strategy is required.

In order to implement an appropriate risk-benefit analysis of such a strategy in an individual patient it is necessary to identify and define the disease in which progression is already occurring although still at a microscopic level. Biological (genomic) progression needs to be identifiable even if the patient is asymptomatic (as useless a criterion in COVID as in neoplasia) and not in radiological progression. In addition to the identification of the target through SSA-PET/CT (current or prior to recent therapy, e.g., surgery), a positive $\left[{ }^{18} \mathrm{~F}\right]-$ FDG PET scan can identify more aggressive forms of disease, which should be considered for early PRRT. Similarly, the blood-based multianalyte NET-related transcriptomic signature, the NETest, which can detect microscopic disease and anticipates imaging progression by up to a year, can be used to early identify the best moment to intervene. In these patients, the blood molecular genomic positive PPQ can stratify such patients into responders and non-responders, so that treatment can be further individualized.

Sadly, humans are reluctant to learn from history and are as incapable of applying the lessons of history to the future. How quickly we forget the bitter fruits of SARS (2002), and now less than two decades later are learning (once again) the extraordinary human costs engendered by the procrastinative errors of COVID-19. Now is the time to better understand the conceptual similarity and better manage the human cancer cell pandemic semantically comparable to NET metastatic disease. Key elements will be early identification, genomic testing, functional imaging (Ga-SSA and FDG PET), and early targeting with an effective agent. Globally embracing these steps and ensuring that an abundance of caution and a desire to extend life are fundamental goals will enable us to advance therapeutic efficacy and succeed. It is our moral and ethical obligation to surmount the unremitting internal pandemic of cancer for the sake of humanity.

In 1721 Pietro Metastasio wrote the memorable Gli orti esperidi, which was immortalized by the "surgical soprano" Carlo Farinelli. In this time of loss, we should follow Metastasio's invitation and facilitate the introduction of PRRT to the Garden of the Hesperides.

\section{Compliance with ethical standards}

Conflict of interest G. Paganelli: None.

L. Bodei: Unpaid consultant for AAA, Ipsen, Clovis, Curium. Research Grant from AAA.

I. Modlin: Medical consultant Clifton LLC.

Ethics approval Not applicable.

\section{References}

1. Strosberg J, Wolin E, Chasen B, Kulke M, Bushnell D, Chaplin M, et al. O-009 NETTER-1 phase III: efficacy and safety results in patients with midgut neuroendocrine tumors treated with $177 \mathrm{Lu}-$ dotatate. Ann Oncol. 2016;27:ii121. https://doi.org/10.1093/ annonc/mdw198.09.

2. Strosberg J, El-Haddad G, Wolin E, Hendifar A, Yao J, Chasen B, et al. Phase 3 trial of (177)Lu-dotatate for midgut neuroendocrine tumors. N Engl J Med. 2017;376:125-35. https://doi.org/10.1056/ NEJMoa1607427.

3. Krenning EPKP, Bakker WH, Breeman WA, Postema PT, Kwekkeboom DJ, Oei HY, et al. Radiotherapy with a radiolabeled somatostatin analogue, [111In-DTPA-D-Phe1]-octreotide. A case history. Ann N Y Acad Sci. 1994;15:496-506.

4. Paganelli GZS, Cremonesi M, Bodei L, Ferrari M, Grana C, Bartolomei M, et al. Receptor-mediated radiotherapy with $90 \mathrm{Y}-$ DOTA-D-Phe1-Tyr3-octreotide. Eur J Nucl Med. 2001;28:42634.

5. Kwekkeboom DJ, Bakker WH, Kam BL, Teunissen JJ, Kooij PP, de Herder WW, et al. Treatment of patients with gastro-enteropancreatic (GEP) tumours with the novel radiolabelled somatostatin analogue [177Lu-DOTA(0),Tyr3]octreotate. Eur J Nucl Med Mol Imaging. 2003;30:417-22. https://doi.org/10.1007/s00259-0021050-8.

6. Bodei L, Cremonesi M, Grana CM, Fazio N, Iodice S, Baio SM, et al. Peptide receptor radionuclide therapy with $(1)(7)(7) \mathrm{Lu}-$ DOTATATE: the IEO phase I-II study. Eur J Nucl Med Mol 
Imaging. 2011;38:2125-35. https://doi.org/10.1007/s00259-0111902-1.

7. Bodei L, Kidd MS, Singh A, van der Zwan WA, Severi S, Drozdov IA, et al. PRRT genomic signature in blood for prediction of (177)Lu-octreotate efficacy. Eur J Nucl Med Mol Imaging. 2018;45:1155-69. https://doi.org/10.1007/s00259-018-3967-6.

8. Bodei L, Kidd MS, Singh A, van der Zwan WA, Severi S, Drozdov IA, et al. PRRT neuroendocrine tumor response monitored using circulating transcript analysis: the NETest. Eur J Nucl Med Mol Imaging. 2020;47:895-906. https://doi.org/10.1007/s00259-01904601-3.

9. Bahri H, Laurence L, Edeline J, Leghzali H, Devillers A, Raoul JL, et al. High prognostic value of $18 \mathrm{~F}-\mathrm{FDG}$ PET for metastatic gastroenteropancreatic neuroendocrine tumors: a long-term evaluation. J Nucl Med. 2014;55:1786-90. https://doi.org/10.2967/ jnumed.114.144386.

10. Severi SNO, Bodei L, Sansovini M, Ianniello A, Nicoletti S, Scarpi $\mathrm{E}$, et al. Role of $18 \mathrm{FDG}$ PET/CT in patients treated with $177 \mathrm{Lu}-$ DOTATATE for advanced differentiated neuroendocrine tumours. Eur J Nucl Med Mol Imaging. 2013;40:881-8.

11. Pavel M, O'Toole D, Costa F, Capdevila J, Gross D, Kianmanesh $\mathrm{R}$, et al. ENETS consensus guidelines update for the management of distant metastatic disease of intestinal, pancreatic, bronchial neuroendocrine neoplasms (NEN) and NEN of unknown primary site. Neuroendocrinology. 2016;103:172-85. https://doi.org/10.1159/ 000443167.

12. NCCN. NCCN Clinical Practice Guidelines in Oncology (NCCN Guidelines $\left.{ }^{\circledR}\right)$, Neuroendocrine and adrenal tumors, Version 1.2019 - March 5, 2019. p. https://www.nccn.org/professionals/physician gls/pdf/neuroendocrine.pdf.
13. Capdevila J, Hernando J, Perez-Hoyos S, Roman-Gonzalez A, Grande E. Meta-analysis of randomized clinical trials comparing active treatment with placebo in metastatic neuroendocrine tumors. Oncologist. 2019;24:e1315-e20. https://doi.org/10.1634/ theoncologist.2018-0675.

14. Paganelli G, Sansovini M, Nicolini S, Grassi I, Ibrahim T, Amadori $\mathrm{E}$, et al. 177Lu-PRRT in advanced gastrointestinal neuroendocrine tumors: 10-year follow-up of the IRST phase II prospective study. Eur J Nucl Med. 2020. https://doi.org/10.1007/s00259-020-04873-0

15. Hudis C, Dang C. The development of dose-dense adjuvant chemotherapy. Breast J. 2015;21:42-51. https://doi.org/10.1111/tbj. 12364.

16. Hill RP. The changing paradigm of tumour response to irradiation. Br J Radiol. 2017;90:20160474. https://doi.org/10.1259/bjr. 20160474.

17. Sansovini M, Severi S, Ianniello A, Nicolini S, Fantini L, Mezzenga E, et al. Long-term follow-up and role of FDG PET in advanced pancreatic neuroendocrine patients treated with (177)LuD OTATATE. Eur J Nucl Med Mol Imaging. 2017;44:490-9. https://doi.org/10.1007/s00259-016-3533-z.

18. Bodei L, Kidd M, Baum RP, Modlin IM. PRRT: defining the paradigm shift to achieve standardization and individualization. J Nucl Med. 2014;55:1753-6. https://doi.org/10.2967/jnumed.114. 143974.

Publisher's note Springer Nature remains neutral with regard to jurisdictional claims in published maps and institutional affiliations. 